\title{
Durch die Gänge der Fabriken läuft der Stundenzähler
}

\author{
Prof. DR.-ING. BERND NOCHE, \\ M.SC.LOGISTIC ENGINEER SERPIL KOC \\ UNIVERSITÄT DUISBURG-ESSEN, INSTITUT FÜR PRODUKT ENGINEERING, TRANSPORTSYSTEME UND -LOGISTIK
}

\section{Zusammenfassung}

Der Lehrstuhl für Transportsysteme und -logistik der Universität Duisburg-Essen befasst sich derzeit mit einem Forschungsprojekt in der Intralogistik. Es handelt sich dabei um das Themengebiet „Einsatz von Flurförderzeugen“. Ziel der Untersuchung ist es, die Ermittlung der Einsatzzeit der Fahrzeuge systematisch zu erfassen und darzustellen. Die Messung der Einsatzzeit hat eine große Bedeutung, wenn es um die Berechnung der Nutzungsdauer und damit um die Kostenabrechnung bei bestimmten Miet-, Leasing oder auch Wartungsverträgen geht. Darüber hinaus dient die Einsatzzeit auch als Berechnungsgrundlage zur Dimensionierung von Fahrzeugflotten zur Bewältigung logistischer Transportaufgaben. Die Ermittlung der Einsatzzeit der Flurförderzeuge erfolgt auf verschiedene Art und Weise - je nach Hersteller oder Vertragsform. Dieses Dokument erläutert die Ergebnisse von einem Fragebogen zur Messung der Einsatzzeit. Für die weitere Informationen und die Präsentation von den Ergebnissen, besuchen Sie bitte folgende Webseite http://www.uni-duisburg-essen.de/tul/

\section{Abstract}

The institute of Transportationsystems and -logistics, University of Duisburg-Essen deals at present with a research-project in the area of Intralogistics. Our research concerns with the following topic "Use of Industrial Trucks". The goal of our investigation is to describe and demonstrate a systematical evaluation of the use-time (hour meter) of the industrial trucks. The measurement way of the hour meter has a high importance, as it has a big influence on the calculation of the lifetime and therewith on the calculation of costs with rent -, leasing and also maintenance contracts. In addition, the use-time is also used as a calculation-basis for the dimensioning from fleets to the overcoming of logistical transportation tasks. The measurement of the use-time of an industrial truck can be calculated in different ways - depending on manufacturers or contract forms. This document illustrates the results of a questionnaire about the measurement of the use-time. For further information and the presentation of the results please visit our web page http://www.uni-duisburg-essen.de/tul/

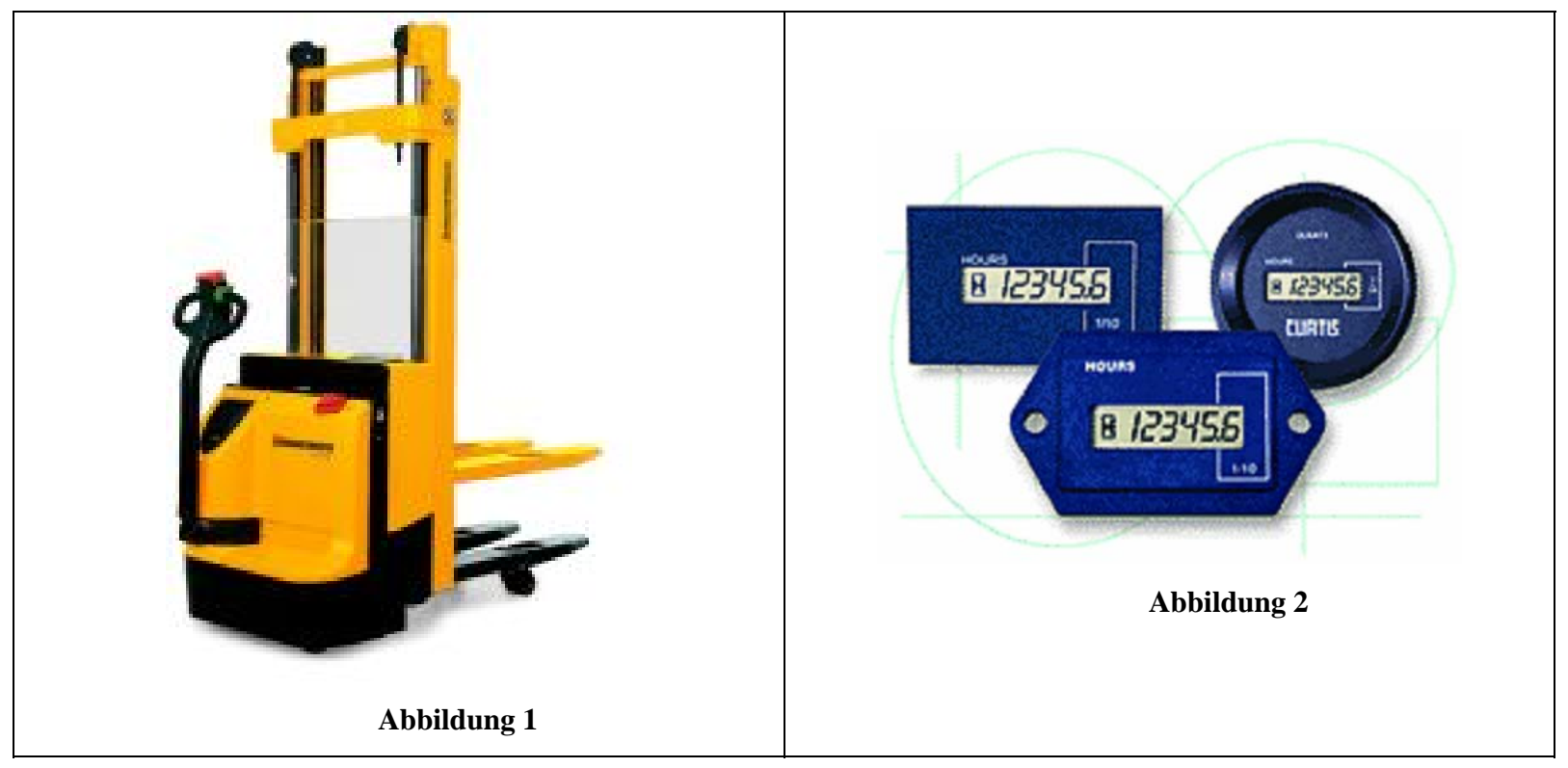


Die Materialtransportsysteme nehmen eine herausragende Stellung in der gesamten Wertschöpfungskette ein. Der wirtschaftliche Erfolg eines Unternehmens hängt u. a. von der effizienten und effektiven Nutzung der Gabelstapler ab. Diese Leistung lässt sich nur mit standardisierten Kennzahlen bzw. Messgrößen bestimmen. Die Art der Erfassung der Einssatzdauer hat weitreichende Auswirkungen auf die technisch-wirtschaftliche Nutzung der Gabelstapler. Die Nutzer und Hersteller müssen die Wirkungen kennen um ihre Betriebsprozesse optimal gestalten zu können.

Der Lehrstuhl für Transportsysteme und -logistik der Universität Duisburg-Essen arbeitete vor kurzem an einem entsprechenden Forschungsprojekt in der Intralogistik. Ziel der Untersuchung war es, die Ermittlung der Einsatzzeit der Fahrzeuge systematisch zu erfassen und die Konsequenzen darzustellen. Die führenden Gabelstapler-Hersteller zeigten ein großes Interesse an diesem Thema und reagierten sehr positiv. Die folgenden Hersteller sind in der Untersuchung einbezogen worden: Atlet, Buescher, BT, Clark, Crown, Jungheinrich, Linde, Nissan-Gabelstapler, OM Pimespo, Still, Toyota, Willecke, Yale und Zeppelin.

Der Stundenzähler erfasst die Stunden, die ein Gabelstapler im Betrieb ist. Die Messung der Einsatzzeit hat eine große Bedeutung, wenn es um die Berechnung der Nutzungsdauer geht. Die Nutzungsdauer fließt in die Kostenabrechnung bei bestimmten Miet-, Leasing oder auch Wartungsverträgen sowie der Ermittlung des Restwertes ein. Durch die falsche Ermittlung der Nutzungsdauer kann ein Gefährdungspotenzial für die Mitarbeiter entstehen, da Instandhaltungstätigkeiten von der Nutzungsdauer abhängen.

Die Gesamtkosten eines Gabelstaplers bezogen auf seine Lebensdauer verteilen sich zu 20\% auf die Anschaffungskosten und zu 80\% auf die Betriebskosten [[Link 1]. Die herstellerspezifischen Messmethoden für die Einsatzzeit führen zu: Informationsmangel über den aktuellen Zustand des Gabelstaplers, ineffiziente Prozesse für die Wartungsplanung, geringere Wartungskontrolle der Ausrüstung, und tragen bei zu: höheren Ausfallzeiten, höheren Wartungskosten mit reduzierter Produktivität und vor allem erschweren Vergleich und Einschätzung von Wartungskosten und anderen Kennzahlen. Deshalb müssen die Betreiber diese Unterschiede zwischen den einzelnen Meßmethoden beachten.

Die Ermittlung der Einsatzzeit der Flurförderzeuge erfolgt auf verschiedene Art und Weise je nach Hersteller oder Vertragsform. Folgende Meßmethoden der Einsatzzeit werden angewandt: Schlüsselzeit - wenn der Gabelstapler eingeschaltet wird (unabhängig von der Nutzungsart), Arbeitsstunden - wenn einer der Motoren läuft, Motorfahrtstunden -wenn der Antriebsmotor läuft, Motorhubstunden - wenn der Hubmotor läuft, Laufstunden - ergibt sich aus der Motorbetriebszeit addiert mit einer festen Zeitspanne oder PalettenBewegungen pro Schicht.

Nach den Ergebnissen der Umfrage stimmten die führenden Gabelstapler-Hersteller darin überein, dass es keine standardmäßigen Meßmethoden der Einsatzzeit gibt. Dies bedeutet, dass es von den einzelnen Herstellern abhängt, wie der Stundenzähler arbeitet. Kalkulationsgrundlage für Transportsysteme sind „die Arbeitsstunden“. Die „Arbeitsstunden“ stellen derzeit die wirtschaftlichste Lösung für den Betreiber dar und sind der beste Weg um das Aktivitätsniveau eines Gabelstaplers zu überwachen. Die Gabelstapler-Hersteller müssen sich der Problematik stellen, die aus den unterschiedlichen Bemessungsgrundlagen für die Gerätenutzung entstehen, um ihre Kundenzufriedenheit steigern zu können. Außerdem betrachten alle Gabelstapler-Hersteller die „Schlüsselzeit“ als die „Arbeitsstunden“ der Verbrennungsmaschinen, weil der Verbrennungsmotor laufend arbeitet.

Die vielfältigen Interpretationsmöglichkeiten der unterschiedlichen Meßmethoden für die Einsatzzeit, müssen die Aufmerksamkeit der Betreiber erregen, da diese wirtschaftliche Folgen für den Nutzer nach sich ziehen. Beinhaltet ein Leasingvertrag eines Herstellers 1000 Betriebsstunden, entspricht es nicht unbedingt dem gleichen Leistungsangebot auf der Basis von Arbeitsstunden eines anderen Herstellers. Die Betreiber sollten die Hersteller fragen, welche Meßmethode benutzt wird. Vor dem Vertragsabschluss soll vereinbart werden, dass im Vertrag beispielsweise „die Arbeitsstunden“ als Grundlage dienen. Kosten pro Stunde, kann durch andere Einsatzzeit-Meßmethoden wesentlich verzerrt werden. Aber am Ende haben die Betreiber das wahre Problem und sie müssen Äpfel mit Äpfeln vergleichen. Die Messung den Arbeitsstunden bedeutet, dass Serviceintervalle länger werden - welche wieder die gesamten Servicekosten reduzieren, wenn man sie mit anderen Meßmethoden z.B. der Laufzeit oder der Schlüsselzeit vergleicht. Wenn ein Lieferant „Laufstunden“ als eine Basis für die Ladung der Batterien nutzt, können sich die Kosten wesentlich erhöhen. Wie nachfolgend in der Grafik gezeigt, es ist überhaupt nicht akzeptabel für die Betreiber, diese negativen Auswirkungen wegen der unterschiedlichen Bemessungsgrundlagen in Kauf zu nehmen. Auch wenn die Kosten für die vereinbarten 1000 Stunden bei den Herstellern gleich sind, können die wahren Kosten für die Betreiber höher sein. Das heißt, der Preis pro Stunde ist gleich aus der Sicht der Anwender, aber der Gabelstapler wird 1000 Stunden auf der Basis von SchlüsselzeitStunden schneller erreichen. Dies bedeutet, dass bei einem Vertrag über 1000 Stunden, die Wartungskosten auf der Basis der Schlüsselzeit z. B. 7.540 € betragen, wohingegen auf der Basis von Arbeitsstunden die Kosten nur $2000 €$ betragen. Basierend auf praxisnahen Zahlen bezogen auf einen Kommissionierstapler kann der Unterschied den Kosten durch die Meßmethode leichter anhand einer Grafik erläutert werden. 


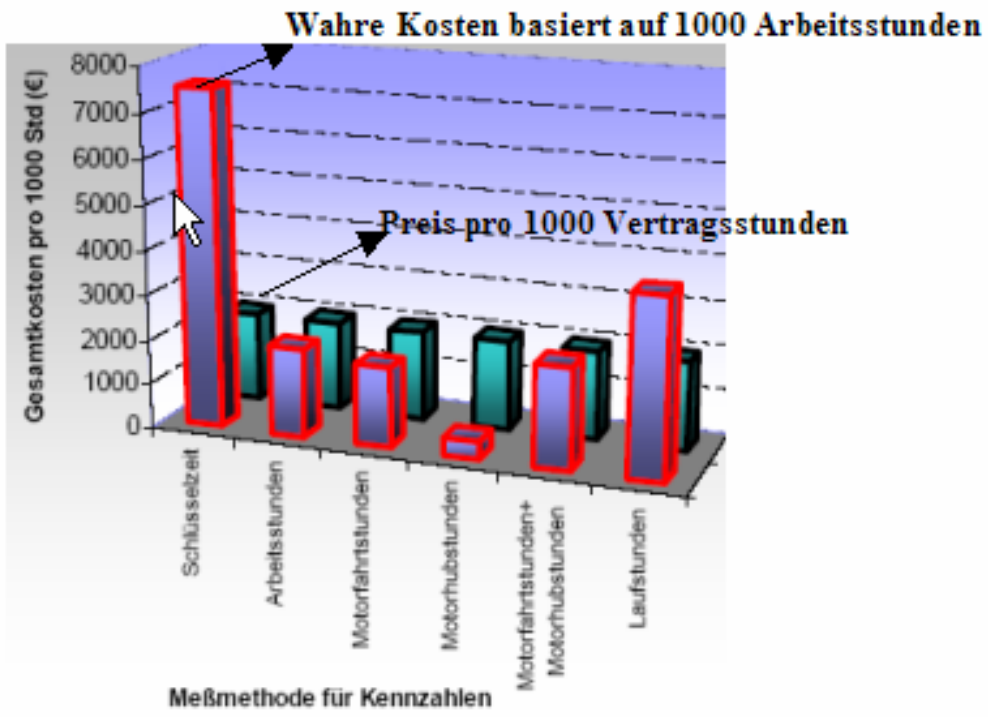

Abbildung 1: Kostenunterschiede die sich lediglich durch die Meßmethode ergeben

Nach den Ergebnissen der Umfrage haben ein Drittel der Hersteller die Meßmethode der Einsatzzeiten und die Konsequenzen auf die Kosten erforscht. Meistens geben die Gabelstapler-Hersteller die Meßmethode ihren Kunden vor und garantieren eine Lebensdauer zwischen 10.000 bis 15.000 Betriebsstunden. Es wird angenommen, dass der Gabelstapler eine wirtschaftliche Nutzungsdauer von acht Jahren besitzt und seine Auslastung durchschnittlich $60 \%$ beträgt. Zusätzlich, fand in den USA das United States Department of the Interior Bureau of Land Management (BLM) in einer Studie heraus, dass industrieweit die durchschnittliche Lebensdauer für industrielle Gabelstapler bei 11.200 Stunden (30. Juni 2003) liegt [Link 2].

Arbeitsstunden sind die Entscheidungsgrundlage für die Bestimmung des Endes des wirtschaftlichen Lebens eines Gabelstaplers. Da es für die Betreiber sehr wichtig ist, gibt es eine Notwendigkeit zur Zusammenarbeit zwischen den Gabelstapler-Herstellern, sie müssen zu einer einvernehmlichen Vereinbarung über die „Definition der Arbeitsstunden“ kommen. Es gibt weitere Forschungen, die noch gemacht werden sollten, um weitere Nutzen herauszufinden. Die Universität Duisburg-Essen unterstützt und fokussiert dieses Thema, denn es gilt das Sprichwort: "Was man nicht messen kann, kann man auch nicht managen“.

\section{Quellenverzeichnis:}

Internet:

\begin{tabular}{|l|l|}
\hline [Link 1] & Johnson Lift, http://www.johnson-lift.com/draft2/index.php?C=fleet_management_service \\
\hline [Link 2] & http://www.sanjoseca.gov/auditor/AuditReports/0311/0311report.pdf \\
\hline
\end{tabular}

Abbildungen

\begin{tabular}{|l|l|}
\hline [Abbildung 1] & http://www.curtisinst.com \\
\hline [Abbildung 2] & http://www.jungheinrich.de \\
\hline
\end{tabular}

